\title{
Trends in Observation-prone Emergency Department Visits Among Michigan Children, 2007-2011
}

\author{
Michelle L. Macy, MD, MS, Lisa Cohn, MS, and Sarah J. Clark, MPH
}

\begin{abstract}
Objectives: To the best of the authors' knowledge, admission of children under observation status in community hospitals has not been examined. The hypothesis of this study was that there has been an increase in observation charge code use over time and variations in the application of observation charge codes across hospital types.
\end{abstract}

Methods: This was a cross-sectional analysis of 5 years (2007 through 2011) of administrative claims data from Michigan residents enrolled in Medicaid, Blue Cross/Blue Shield of Michigan preferred provider organization, and Blue Cross Network health maintenance organization compiled into a single data set. Emergency department (ED) visits to facilities in Michigan made by children (younger than 18 years) were selected. Observation-prone ED visits were identified based on the presence of International Classification of Diseases, Ninth Revision, Clinical Modification (ICD-9-CM) codes. Counts of observationprone ED visits were determined and descriptive statistics were calculated. Changes over time in the proportion of visits with observation charge codes by hospital type were assessed with chi-square analysis.

Results: The observation-prone ICD-9-CM codes were identified in 881,622 ED visits made by children to 142 Michigan facilities during the 5 -year study period. Overall, the vast majority of visits $(n=646,499$; $91.0 \%$ ) with the selected ICD-9-CM codes resulted in discharge from the ED without associated observation or inpatient charge codes. Among the 64,288 visits that resulted in admission for observation or inpatient care, observation charge codes without inpatient charge codes were applied to 22,933 $(35.7 \%)$ admissions, observation and inpatient charge codes were applied to 4,756 (7.4\%) admissions, and inpatient charge codes without observation charge codes were applied to 36,599 (56.9\%) admissions. Hospitals with pediatric ED and inpatient services (Type 1 and Type 2 hospitals) had higher proportions of ED visits that went on to admission for observation or inpatient care (15.9 and 10.7\%) than hospitals without pediatric ED services (Type 3 and Type 4 hospitals; 7.2 and 3.7\%). The proportion of admissions that had observation charge codes for all hospital types increased over time, most prominently among Type 1 and Type 2 hospitals.

Conclusions: The application of observation charge codes to Michigan children with observation-prone conditions has increased over time across all hospital types. There is a need to evaluate pediatric observation care in diverse settings to compare the effectiveness of different models.

ACADEMIC EMERGENCY MEDICINE 2015;22:483-486 @ 2015 by the Society for Academic Emergency Medicine

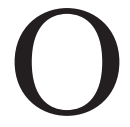

ne in three U.S. children hospitalized in 2003 experienced a short stay, with discharge on the day of, or day after, admission. ${ }^{1}$ Between 2004 and 2009, a growing proportion of short-stay hospital- izations in children's hospitals were admitted under "observation status," an administrative designation assigned when patients do not meet hospital or payer criteria for inpatient care. ${ }^{2}$ To the best of our

From the Department of Emergency Medicine, Children's Emergency Services (MLM), Child Health Evaluation and Research (CHEAR) Unit, Division of General Pediatrics (MLM, LC, SJC), University of Michigan, Ann Arbor, MI.

Received August 14, 2014; revision received October 16, 2014; accepted October 17, 2014.

This project was supported through a grant from the Center for Healthcare Research and Transformation.

Dr. Macy, an associate editor for this journal, had no role in the peer-review process or publication decision for this paper.

The authors have no potential conflicts to disclose.

Supervising Editor: Marc H. Gorelick, MD, MSCE.

Address for correspondence and reprints: Michelle L. Macy, MD, MS; e-mail: mlmacy@umich.edu. 
knowledge, admission of children under observation status in community hospitals has not been examined.

We analyzed 5 years of administrative claims data from Michigan Medicaid, Blue Cross/Blue Shield of Michigan (BCBSM), and Blue Care Network (BCN), to understand the application of observation charge codes to children experiencing emergency department (ED) visits for conditions that are commonly admitted under observation status ${ }^{2}$ and have been successfully treated in physically distinct pediatric observation units. ${ }^{3} \mathrm{We}$ hypothesized an increase in observation charge code utilization over time and variation in the application of observation charge codes across hospital types.

\section{METHODS}

\section{Study Design}

This was a cross-sectional analysis of 5 years (2007 through 2011) of administrative claims data from Michigan residents enrolled in Medicaid, BCBSM preferred provider organization, and the BCN health maintenance organization compiled into a single data set. The University of Michigan Medical School Institutional Review Board approved this study.

\section{Study Setting and Population}

Emergency department visits to facilities in Michigan made by children ( $<18$ years) were selected based on the presence of ED revenue codes (045x). We identified observation-prone ED visits based on the presence of International Classification of Diseases, Ninth Revision, Clinical Modification (ICD-9-CM) codes (Data Supplement S1, available as supporting information in the online version of this paper).

\section{Study Protocol}

The ICD-9-CM codes were chosen to be representative of conditions that are commonly admitted under observation status ${ }^{2}$ and have been successfully treated in physically distinct pediatric observation units, ${ }^{3}$ including allergic reaction, cellulitis, dehydration, diabetic ketoacidosis, head injury, headache, ingestion, respiratory conditions, and seizure. Continuous enrollment for the 90 days before and after an ED visit was required for study eligibility.

Emergency department visits were excluded based on the presence of ICD-9-CM codes indicative of severe illness or complex comorbid conditions in claims related to the ED visit (Data Supplement S2, available as supporting information in the online version of this paper), because these patients are generally considered inappropriate for observation care. ED visits resulting in transfer out of the ED were excluded due to the inability to link outcomes for the episode of care across different settings on the same billing date. Visits with ED length of stay of three nights or more without observation or inpatient admission were excluded due to the likelihood of administrative data errors. Visits with negative values for ED length of stay were also excluded.

\section{Measures}

ED Visit Outcome. Disposition from the ED was categorized into four groups based on the presence of observation and inpatient charge codes: discharged from the ED, observation only, observation plus inpatient, inpatient only.

Hospital Characteristics. Hospitals were grouped into four categories: Type 1, children's hospital; Type 2, general hospital with pediatric medical-surgical beds and a pediatric ED; Type 3, general hospital with pediatric medical-surgical beds without mention of a pediatric ED; and Type 4, hospital without mention of pediatric-specific care. These distinctions were based on membership in the National Association of Children's Hospitals and Related Institutions and information contained in the 2012 American Hospital Association Guide to the Health Care Field ${ }^{4}$ and the 2012 Michigan College of Emergency Physicians Emergency Department Directory. ${ }^{5}$ Twenty-seven hospitals without published information about pediatric services were considered undetermined.

\section{Data Analysis}

Counts of observation-prone ED visits were determined and descriptive statistics were calculated. The proportions of visits for each disposition category were calculated for the overall sample and by hospital type. Changes in the proportion of admissions with observation charge codes over the 5 years of study were assessed using chi-square statistics. All analyses were performed using SAS version 9.3.

\section{RESULTS}

The observation-prone ICD-9-CM codes were identified in 881,622 ED visits made by children to 142 Michigan facilities during the 5-year study period. The ED visits with the selected ICD-9-CM codes represented about one-quarter of all ED visits to Michigan facilities for children covered by Michigan Medicaid, BCBSM, or BCN. The final analytic sample included 710,787 ED visits, with exclusions for lack of continuous enrollment ( $n=102,536)$, severe illness or complex comorbid condition $(n=42,687)$, transfer $(n=25,265)$, and length of stay outliers $(n=347)$.

The study population consisted of 68,535 (9.6\%) children less than 1 year; 158,501 (22.3\%) children 1 and 2 years; 95,232 (13.4\%) children 3 and 4 years; 232,354 $(32.7 \%)$ children 5 through 12 years; and 156,165 $(22.0 \%)$ children 13 through 17 years. Over half of visits were made by males ( $n=396,268 ; 55.8 \%$ ), and children covered by Medicaid ( $n=558,385 ; 78.6 \%$ ). The greatest number of visits ( $n=271,241 ; 38.2 \%$ ) occurred in the 57 Type 3 hospitals; 188,207 (26.5\%) visits occurred in the six Type 1 hospitals; $172,935(24.3 \%)$ visits occurred in the 44 Type 4 hospitals; 60,035 (8.4\%) visits occurred in the eight Type 2 hospitals; and 18,369 (2.6\%) visits occurred in the 27 hospitals of undetermined type.

Overall, the vast majority of visits ( $n=646,499$; $91.0 \%$ ) with the selected ICD-9-CM codes resulted in discharge from the ED without associated observation or inpatient charge codes. Type 1 and Type 2 hospitals had higher proportions of ED visits that went on to admission for observation or inpatient care (15.9 and $10.7 \%$ ) than Type 3 and Type 4 hospitals (7.2 and $3.7 \%$ ). 
Hospital Type 1

Children's Hospitals, $n=6$

Total ED Visits, $n=188,207$

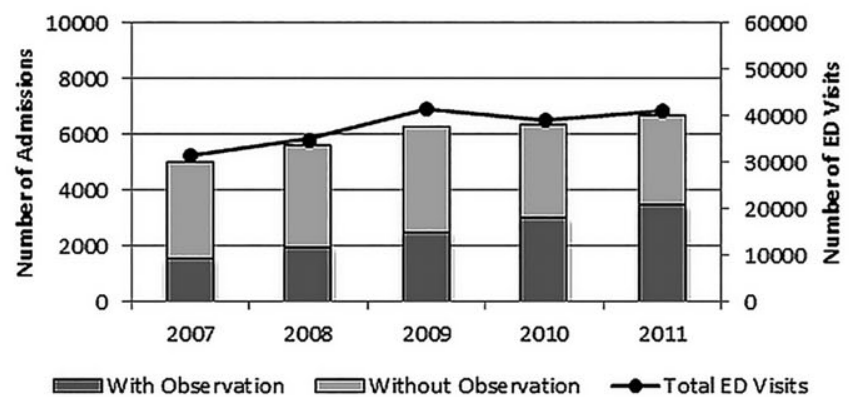

Hospital Type 3

Hospitals with Pediatric Inpatient Services, $n=57$ Total ED Visits, $n=271,241$

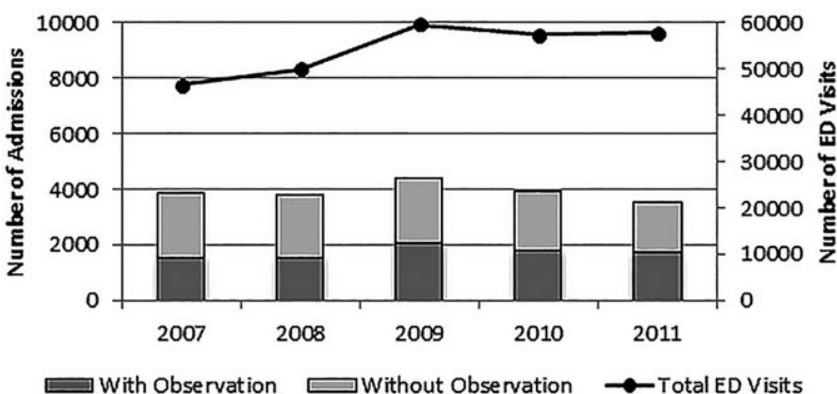

Hospital Type 2

Hospitals with Pediatric Inpatient and ED, $n=8$

Total ED Visits, $\mathrm{n}=60,035$

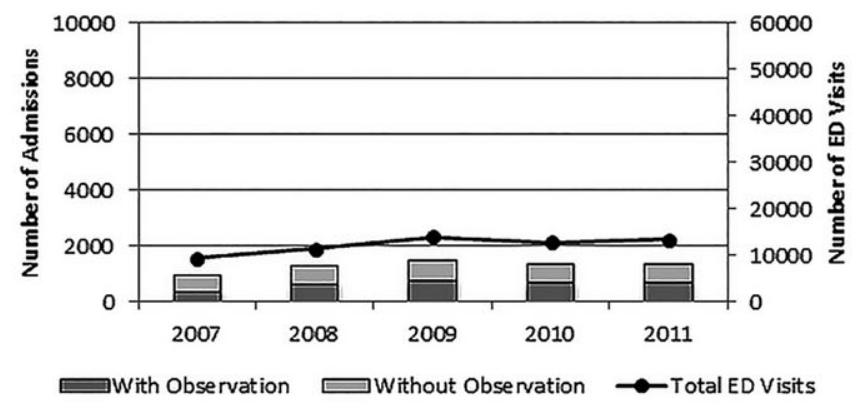

Hospital Type 4

No Specific Pediatric Services, $n=44$

Total ED Visits, $n=172,935$

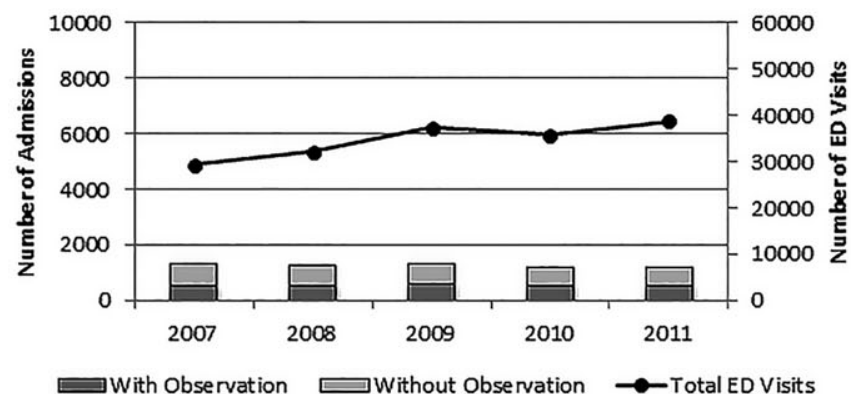

Figure 1. Trends in admissions with and without observation charge codes and total ED visits over the 5-year study period by hospital type. The stacked bar graph on the primary axis in each panel represents ED admissions. The dark gray bars indicate admissions with observation charge codes (with and without inpatient charge codes) in each year for each hospital type. The light gray bars indicate admissions without observation charge codes (i.e., inpatient charge codes alone) in each year for each hospital type. The line graph on the secondary axis in each panel represents the total number of ED visits (admissions and discharges) in each year for each hospital type. The ranges for the primary and secondary axes are the same across panels to allow for comparisons between hospital types.

Among the 64,288 visits that resulted in admission for observation or inpatient care, observation without inpatient charge codes were applied to 22,933 (35.7\%) admissions, observation and inpatient charge codes were applied to $4,756(7.4 \%)$ admissions, and inpatient without observation charge codes were applied to $36,599(56.9 \%)$ admissions.

Five-year trends in ED visits and admissions with and without observation charge codes by hospital type are presented in Figure 1. The proportion of admissions that had observation charge codes for all hospital types increased over time $(\mathrm{p}<0.001)$, most prominently among Type 1 and Type 2 hospitals. Among the 27,689 admissions with observation charge codes in the sample, 4,756 (17.2\%) had associated inpatient charge codes, suggesting that patient status converted from observation to inpatient or inpatient to observation during the stay. Type 1 and Type 2 hospitals had higher proportions of observation admissions with associated inpatient charge codes (19.0 and 20.4\%) than Type 3 and Type 4 hospitals (14.7 and 12.8\%).

\section{DISCUSSION}

Over the 5-year study period, over 700,000 pediatric ED visits made by children covered by Michigan Medicaid,
BCBSM, or BCN had observation-prone diagnoses. While the proportion of visits with observation-prone diagnoses resulting in admission remained steady over time, the use of observation charge codes increased. Because hospitals may be reimbursed at lower rates for care provided to patients under observation status compared with patients who meet inpatient status, ${ }^{6}$ these trends may result in pressures on emergency physicians to avoid admission and extend ED treatment durations for children under observation status.

This is the first study to our knowledge to examine the application of observation charge codes to pediatric ED visits outside of freestanding children's hospitals. We found that ED visits for children with observationprone diagnoses were widely distributed across hospitals, ranging from large freestanding children's hospitals to small community hospitals. Pediatric observation status patients in children's hospitals typically receive care in inpatient settings, ${ }^{7}$ with resource utilization that is comparable to patients with similar diagnoses admitted as inpatients. ${ }^{8}$ Several single-center studies suggest that children with select diagnoses can be treated effectively in pediatric observation units with potential for shorter length of stay and costs savings. ${ }^{3}$ Yet pediatric observation units are present in $39 \%$ of freestanding children's hospitals, ${ }^{7} 39 \%$ of hospitals with 
separate pediatric wards, ${ }^{9}$ and just $4 \%$ of hospitals without pediatric wards. $^{9}$ Our results indicate a growing population of pediatric ED patients who may benefit from the development of cost-effective alternatives to inpatient care that draw on the principals of observation medicine. ${ }^{10}$

\section{LIMITATIONS}

First, we focused on specific ICD-9-CM codes that were based on a set of diagnosis groups from the literature. While these diagnoses overlap with many of the conditions treated in pediatric observation units, they do not reflect all conditions that may be suited to observation medicine. Thus, we likely underestimated the number of observation-prone ED visits. Conversely, the limited set of exclusionary diagnoses likely resulted in the inclusion of some children who were too sick or medically complex for observation care. Second, our method for categorizing hospitals did not include information about pediatric observation services (e.g., physically distinct vs. virtual unit; located in the ED or inpatient setting); therefore, we cannot draw conclusions about which models of care are most commonly used for the treatment of children with observation-prone diagnoses. Finally, our results from Michigan may not be generalizable to other states.

\section{CONCLUSIONS}

The application of observation charge codes to Michigan children experiencing ED visits for observation-prone conditions has increased over time across all hospital types. By 2011, nearly half of all admissions from the ED for observation-prone conditions had associated observation charge codes. There is a need to evaluate pediatric observation care in diverse settings to compare the effectiveness of different models for pediatric observation care.

\section{References}

1. Macy ML, Stanley RM, Lozon MM, Sasson C, Gebremariam A, Davis MM. Trends in high-turnover stays among children hospitalized in the United States, 1993-2003. Pediatrics 2009;123:996-1002.

2. Macy ML, Hall M, Shah SS, et al. Pediatric observation status: are we overlooking a growing population in children's hospitals? J Hosp Med 2012;7:530-6.

3. Macy ML, Kim CS, Sasson C, Lozon MM, Davis MM. Pediatric observation units in the United States: a systematic review. J Hosp Med 2010;5:17282.

4. American Hospital Association. AHA Guide. Chicago, IL: American Hospital Association, 2011.

5. Michigan College of Emergency Physicians. Michigan Emergency Department Directory. Lansing, MI: Michigan College of Emergency Physicians, 2012.

6. Sheehy AM, Graf B, Gangireddy S, et al. Hospitalized but not admitted: characteristics of patients with "observation status" at an academic medical center. JAMA Intern Med 2013;173:1991-8.

7. Macy ML, Hall M, Shah SS, et al. Differences in designations of observation care in US freestanding children's hospitals: are they virtual or real? J Hosp Med 2012;7:287-93.

8. Fieldston ES, Shah SS, Hall M, et al. Resource utilization for observation-status stays at children's hospitals. Pediatrics 2013;131:1050-8.

9. Middleton K, Burt C. Availability of pediatric services and equipment in emergency departments: United States, 2002-2003. Adv Data 2006;367:1-16.

10. Graff LG. Observation Medicine: The Healthcare System's Tincture of Time. In: Graff LG (editor). Principles of Observation Medicine. Dallas, TX: American College of Emergency Physicians, 2010.

\section{Supporting Information}

The following supporting information is available in the online version of this paper:

Data Supplement S1. International Classification of Diseases, Ninth Revision, Clinical Modification (ICD-9CM) code-based inclusion criteria.

Data Supplement S2. International Classification of Diseases, Ninth Revision, Clinical Modification (ICD-9CM) code-based exclusion criteria. 